\title{
LA CONQUISTA HITITA DE ALAŠIYA
}

\author{
Natalia Lodeiro Pichel \\ (Universidad Autónoma de Madrid)
}

\begin{abstract}
RESUMEN
La influencia del Imperio hitita durante el Bronce Final en el Oriente Próximo era indudable; sin embargo, el declive al que se vio abocado el Imperio durante sus últimas décadas obligó a sus últimos reyes a fijar sus metas, por vez primera, más allá del mar. Las sucesivas conquistas del reino de Alašiya, en la isla de Chipre, intentan paliar una grave situación para el reino que era ya irreversible. En el presente artículo se lleva a cabo un análisis de las relaciones entre el reino de Hatti y Alašiya, antes y después de su conquista.
\end{abstract}

\section{PALABRAS CLAVE}

Hatti, Alašiya, Anatolia, Chipre, Edad del Bronce Final

\begin{abstract}
The influence of the Hittite Empire through the Late Bronze Age in the Near East was certain; nevertheless, the decline of the Empire during its last decades, forced its kings to set their goals, for the first time, beyond the sea. The successive conquests of the kingdom of Alašiya, on the island of Cyprus, sought to alleviate a difficult condition for the Hittite Kingdom, which was already irreversible. This paper presents an analysis of the relations between the kingdom of Hatti and Alašiya, before and after its conquest.
\end{abstract}

\section{KEYWORDS}

Hatti, Alašiya, Anatolia, Cyprus, Late Bronze Age

Las fuentes textuales de los ss. XIII y XIV a.C. procedentes del Próximo Oriente, especialmente en Egipto y Ugarit, pero también de Hatti, mencionan un reino independiente, Alašiya, siendo considerado por sus monarcas como similar en poderes a los suyos propios ${ }^{1}$, con el que tienen tratos comerciales. Parte de las tablillas de Amarna y Ugarit, que atestiguan la correspondencia de ambos y Alašiya, fueron analizadas con el fin de averiguar su procedencia; revelando que la arcilla provenía de la isla de Chipre ${ }^{2}$. Por lo tanto, en la actualidad se acepta que el reino de Alašiya se encontrase en Chipre, pero aún se discute si el reino formaba un poder central en la isla ${ }^{3}$; o si por el contrario la isla se constituía en un conglomerado de reinos regionales ${ }^{4}$.

Las referencias en el corpus hitita sobre Alašiya son escasas y la mayoría pertenecen a las últimas décadas del Imperio ${ }^{5}$. La primera noticia de Alašiya documentada en los textos hititas es en "El Desafuero de Madduwatta" (CTH 147). En este texto, un vasallo hitita, Madduwatta, realizó sucesivas redadas en Alašiya durante el reinado de Arnuwanda

\footnotetext{
1 En la correspondencia entre Alašiya y Egipto (EA33-40), se puede comprobar como el faraón y el rey se trataban de hermanos, título reservado a los grandes reyes de la época.

2 Goren et al., 2003.

3 Muhly, 1972; Knapp, 1988; 2008; Webb, 1999.

4 Keswani, 1996; Steel, 2004.

5 Stefanini (1964-1965) opina que la carta escrita por la reina hitita Puduhepa (KUB21.38), tratando la alianza matrimonial de la princesa hitita con un rey extranjero, podría estar destinada al rey de Alašiya; sin embargo, numerosos indicios (Beckman, 1996:125-129; Hoffner, 2009, 281-929) apuntan a que se trata de una de las cartas de la correspondencia entre la reina hitita y el faraón Ramsés II con motivo de la alianza matrimonial entre ambos reinos.
} 
I, ayudado por Attariššiya ${ }^{6}$, descrito como un hombre de Ahhiyiya ${ }^{7}$. El rey hitita Arnuwanda I se lo reprocha, ya que reclamaba su soberanía sobre la isla.

La flota de la que disponía Madduwatta debía de ser de origen Lukka, quien pudo haber ganado de alguna forma el control sobre la región, ya que sus habitantes eran conocidos por sus actividades piratas y sus incursiones en las ciudades costeras de Alašiya se atestiguan décadas más tarde durante el reinado del faraón Akenatón ${ }^{9}$. Si las reclamaciones de Hatti sobre la isla fuesen verídicas, Madduwatta hizo caso omiso de las quejas del rey hitita, ya que el reino de Hatti no disponía de una flota propia y no podría haber ejercido su autoridad sobre el territorio de una forma práctica.

Aparte de esta referencia, que resultó en una simple queja sin ningún resultado aparente; no se conoce ninguna participación política o militar del Imperio hitita hacia Alašiya antes de los reinados de Tudhaliya IV y Suppiluliuma II. Sin duda, la soberanía hitita sobre Alašiya, durante el reinado de Arnuwanda I, debe haber sido ejercida de una manera más simbólica que rea ${ }^{10}$. Este hecho también demostraría que, al menos, durante las primeras décadas del s. XIV a.C. la autoridad ejercida por los hititas en Anatolia occidental parece poco más que simbólica en algunos de los territorios que reclamaban; intentando mantenerla a través de vasallos locales, comprometidos a mantener la lealtad al rey.

Esta falta de autoridad hitita sobre la región brindó la oportunidad para la expansión de las actividades e influencia de los Ahhiyawa, tal vez inicialmente por líderes militares independientes, como Attariššiya, que propiciaron una notable presencia de Ahhiyawa en la región a finales de siglo ${ }^{11}$.

Alašiya era, así mismo, un lugar común para los fugitivos procedentes de varios países del Próximo Oriente durante el Bronce Final que encontraban refugio en la isla ${ }^{12}$. En un texto perteneciente al reinado de Mursili II (KUB XIV 14), el rey hitita escribe acerca de unos asesinatos acontecidos durante el reinado de su padre Suppiluliuma I, en los que varios miembros de la familia real involucrados fueron enviados a Alašiya. También, en la "Apología de Hattusili III" (KUB. I 1), al mencionar un conflicto dentro del seno de la familia real, ocurrido en el reinado de su padre, el rey Mursili II, Hattusili tiene el poder para ejecutar a un hombre llamado Arma-Tarhunta; sin embargo, lo envía a Alašiya ${ }^{13}$. Éstos, y varios textos provenientes de Ugarit ${ }^{14}$ afirmarían que Alašiya era un lugar común para los refugiados, tanto criminales como refugiados políticos, que gozarían de una plena independencia personal en la isla, sin que las autoridades locales los extraditasen; siendo una característica única en el Mediterráneo oriental en el Bronce Final ${ }^{15}$.

La campaña de Tudhaliya IV para conquistar la isla es la primera indicación clara de las ambiciones hititas sobre el territorio desde las reclamaciones del rey Arnuwanda I; debido, principalmente, a las presiones de Aḩhiyawa en el oeste y de Asiria, en el este,

\footnotetext{
6 Collins, 2007: 44.

7 Ahhiya, o Ahhiyawa, se asocia con la Grecia micénica (algunos estudios recientes, entre otros: Beckman et al., 2011; Bryce, 2011; Carruba, 1995; Ünal,1991; Houwink ten Cate, 1983-1984)

8 No está claro si los hititas mantenían una soberanía de facto en la isla pero, si esto fue así, recuperó su independencia muy pronto, ya que Alašiya no se menciona en la lista de aliados hititas en la Batalla de Qadesh.

9 Bryce, 2005: 135

10 Bryce, 2005: 135.

11 Collins, 2007: 44-45.

12 Klengel (1999: 262) apunta a que este reino sirvió como refugio a los nobles hititas durante las luchas dinásticas.

13 Heltzer, 2001:370-371.

14 En una carta del rey de Beruta al rey de Ugarit, éste le pide la devolución de los refugiados en Alašiya, en caso de que volviesen a su reino y, en otro texto, el rey de Karkemish envía dos princesas de Ugarit a Alašiya. 15 Heltzer, 2001:370-372.
} 
quienes les habían arrebatado el control de la zona minera de Ergani Maden, en el sureste de Anatolia. Tudhaliya, por lo tanto, debía asegurarse que las rutas comerciales, de las que dependían para suplir al reino de grano y de materias primas, continuaban accesibles. Esta campaña fue la primera batalla naval hitita de la que se tienen registros; y para ésta, Tudhaliya, sin duda, contaba con el apoyo de sus vasallos de Ugarit ${ }^{16}$ y de Amurru quienes disponían de una flota. Aunque Tudhaliya IV se aseguró, temporalmente, de establecer un régimen prohitita en la isla; sin embargo, su control sobre el reino fue efímero ya que su hijo, Suppiluliuma II, se vio obligado a realizar tres incursiones navales, y otra batalla final en tierra para recuperar el control del reino insular ${ }^{17}$, y de una ruta comercial segura para el reino, que sufría varias hambrunas ${ }^{18}$. Sin embargo, esta victoria resultó igual de efímera que la de su padre, ya que Huatti y Alašiya ${ }^{19}$ fueron invadidos por los Pueblos del Mar.

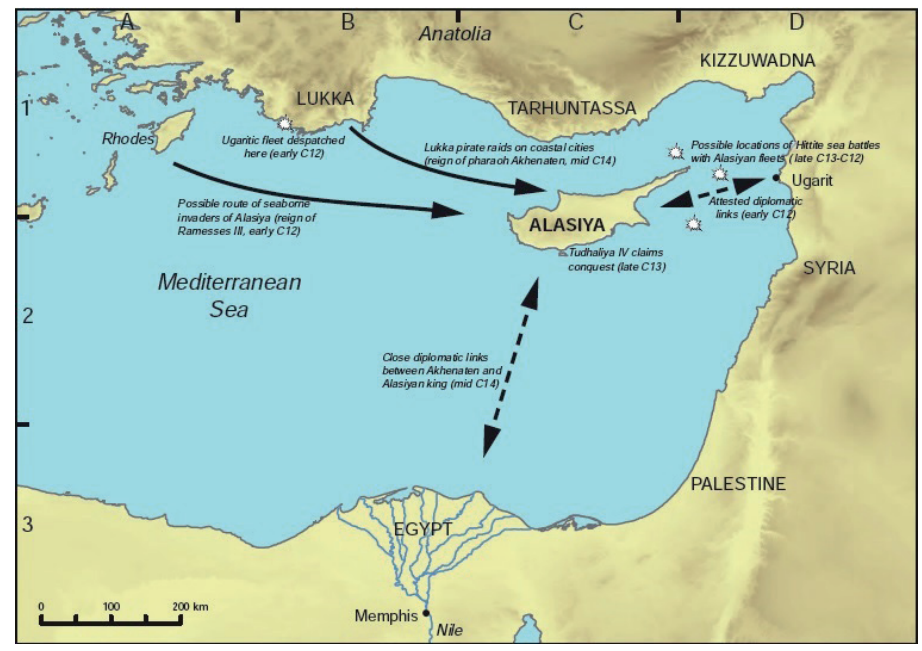

Figura 1: Mapa de Alašiya, con los diferentes ataques de los hititas y otros pueblos invasores. (Bryce, T. y Birkett-Rees, J., 2016, Atlas..., p. 147).

Ambas campañas se describen en la tablilla KBo XII 38, datada en los últimos años del Imperio hitita. Esta tablilla, escrita en cuneiforme, está fragmentada en su parte superior e inferior pero las cuatro columnas se pueden leer casi en su totalidad. Güterbock ${ }^{20}$ sugiere que este texto trata dos batallas diferentes entre Alašiya y Huatti. La primera, descrita en la primera columna, sería la conquista de este reino por el rey hitita Tudhaliya IV y la segunda, en la tercera columna, por su hijo, y sucesor, Suppiluliuma II. Los temas que

\footnotetext{
16 El apoyo naval que prestó Ugarit a los reyes hititas Tudhaliya IV y Suppiluliuma II supuso la desprotección de este reino vasallo. En una carta (RS 18.147) del rey de Ugarit, Ammurapi, pidiéndole asistencia al rey de Alašiya, se constata como el envío de su flota al rey hitita ha dejado desprotegido a su reino, que no puede repeler una invasión.

17 Los enemigos, de los que se desconoce su origen, probablemente eran los Pueblos del Mar (de Martino, 2008: 249-250).

18 La necesidad de los reyes hititas de proteger las rutas comerciales era fundamental desde el reinado de Hattusili III. Una tablilla hitita de este periodo (CTH 165) trata los envíos de grano por parte del faraón egipcio Ramsés II para aliviar la hambruna en Hatti. Otras fuentes, durante el reinado de Tudhaliya IV, constatan que éste recibió en el quinto año de reinado del faraón Merneptah otro cargamento y en una carta al rey de Ugarit le pide que le envíe grano (Bryce, 2005, 331).

19 Ramsés III, en los relieves de Medinet Habu, muestra su victoria sobre los Pueblos del Mar mientras relata como una serie de países, entre ellos Huatti y Alašiya, fueron destruidos. Además, un segundo nivel de destrucción en Enkomi, posiblemente asociado a las destrucciones de los Pueblos del Mar, está datado a finales del LH IIIC Ib. y la ocupación de la isla por los Pueblos del Mar entre 1200 y 1110 a.C. está visible en la estratigrafía (Schaeffer, 1952: 369).

20 Güterbock, 1967:75.
} 
se tratan en la tablilla serían los siguientes ${ }^{21}$ : imposición de un tributo a Alašiya por un rey hitita no identificado (columna I); realización de una estatua de Tudhaliya con una inscripción que relata sus obras y la colocación de ésta en el edificio ${ }_{4}^{\mathrm{NA}}$ hekur SAG.UŠ por su hijo Suppiluliuma II (columna II); una presentación de Suppiluliuma II, seguida de su genealogía y títulos, que termina con las palabras: "mi padre Tudhaliya" (columna III); informe de una batalla naval contra Alašiya por Suppiluliuma II, seguida de otra en tierra (columna III); y por último, una referencia al edificio ${ }_{4}^{\mathrm{NA}}$ hekur SAG.UŠ y a la estatua, seguida por una maldición protectora (columna IV).

Por lo tanto, la primera columna trataría la conquista de Alašiya por el rey Tudhaliya IV y la imposición de un tributo. En la segunda columna, el rey Suppiluliuma II relata que erigió una imagen de su padre, el rey Tudhaliya IV, y la colocó en el edificio ${ }^{\mathrm{NA}}{ }_{4}$ hekur SAG.UŠ que ordenó realizar, terminando su relato con una descripción de su genealogía. La tercera columna trata la derrota y quema de los barcos de Alašiya por Suppiluliuma en el "medio del mar", y la cuarta columna se refiere al edificio ${ }_{4}{ }_{4}$ hekur SAG.UŠ, sus privilegios y sanciones contra quienes abusen de ellos ${ }^{22}$.

El tributo que debía pagar el rey, y el ${ }^{\text {Lú }}$ pidduri $^{23}$, de Alašiya se constituía por cuatro cargamentos iguales destinados a la diosa Sol de Arinna y los dioses de la Tormenta de Zippalanda, Nerik y Hुatti y a su sacerdote, el rey hitita. Cada uno de éstos contenía oro, un talento de cobre y tres seah de gayyātum ${ }^{24}$. El gayyātum era un producto, que se lista junto al pan, la harina y cebada en los textos pertenecientes al II y III milenio a.C. en Mesopotamia, por lo que podría ser, así mismo, un tipo de cereal. Sin embargo, Itamar y Graciela Gestoso Singer ${ }^{25}$ creen que este gayyātum sería un aceite perfumado, un ungüento o incienso que recibe este nombre por uno de sus ingredientes. En su estudio del análisis de las fuentes textuales y arqueológicas, lo identifican como un producto de lujo producido en la isla, que era famosa por su producción de aceites, y exportado a los nobles de las cortes de los diferentes reinos del Próximo Oriente ${ }^{26}$.

En cuanto a la localización de la estatua erigida por el rey Suppiluliuma II, Otten ${ }^{27}$ cree que esta estatua se habría situado en la Cámara B de Yazilikaya, la cámara funeraria del rey Thudaliya IV. Sin embargo, una inscripción de Nisantepe ${ }^{28}$, en la ciudad alta de Hattuša, que se encuentra en mal estado de conservación, comienza: "Yo (soy) Suppiluliuma" seguido de sus títulos y genealogía, continuando con "[Mi padre] Tudhaliya [...”; correspondiéndose, de manera exacta, al texto de la tablilla KBo XII $38^{29}$. Además, el edificio presenta dos fases constructivas: la primera, una rampa que llevaría a la puerta de la esfinge; y la segunda, datada del mismo periodo de la inscripción, que correspondería al reinado de Suppiluliuma II. Por lo tanto este edificio podría haber sido proyectado por el rey Tudhaliya IV, pero construido por su hijo, como se revela en el texto hitita ${ }^{30}$.

La tablilla KBo XII 39 (CTH 141) es un tratado entre Hatti y Alašiya, siendo el único entre ambos reinos del que se tiene noticia. Debido al estado muy fragmentado no se puede conocer el nombre del rey hitita al que pertenece, pero podría ser Tudhaliya IV

\footnotetext{
21 Güterbock, 1967:74-74; Hawkins, 1995:58.

22 Güterbock, 1967:74.

23 Probablemente haya que identificarlo como un gobernador (de Martino, 2008: 255-256).

24 Güterbock, 1967:77-78.

25 Singer y Gestoso Singer, 2014: 327.

26 Singer y Gestoso Singer, 2014: 325-236.

27 Otten, 1963: 22.

28 Güterbock, 1967:81.

29 Hawkins, 1995: 59.

30 van den Hout, 2002: 77-78.
} 
o Suppiluliuma II ${ }^{31}$. El tratado presenta el siguiente contenido ${ }^{32}$ : un prólogo histórico, que no se conserva; las estipulaciones, muy fragmentadas, en donde se observa la obligación de Alašiya de entregar a los prisioneros y la obligación de informar al rey hitita de todos los asuntos que le concerniesen ${ }^{33}$; las bendiciones, juramentos y listas de testigos; y por último, el depósito del documento. Este depósito, ante la diosa Ishtar en Ḩatti y Alašiya, es interpretado por Kenneth A. Kitchen y Paul J. N. Lawrence ${ }^{34}$ como la constatación de que se trababa de una rara anexión al Imperio hitita que el rey querría fijar de todas las maneras, tanto personalmente como públicamente. El depósito del tratado en Alašiya frente a la diosa Isthar, sugiere también una posible veneración a esta diosa en la isla ${ }^{35}$.

El texto reafirma que hasta la conquista de Alašiya por el rey Tudhaliya IV, los refugiados hititas no encontraban ninguna oposición por las autoridades locales a su permanencia ni eran extraditados. Sin embargo, la cláusula presente en este tratado, muestra un cambio en las políticas hititas, al demandar el rey hitita el retorno de los fugitivos y una probable supervisión de los exiliados hititas en la isla ${ }^{36}$.

En otro texto, KBo I 26 (CTH 216), un rey hitita reclama bienes que derivan de la isla. Knapp ${ }^{37}$ lo identifica como una carta, o una copia, en acadio realizada para ser enviada a Alašiya desde la corte hitita. El documento dataría de la segunda mitad del s. XIII, posterior a la conquista de Tudhaliya IV o Suppiluliuma II. En la tablilla KBo I 26 el tributo consiste en oro, rhyta, ceñidores y prendas para los caballos ${ }^{38}$. De estos productos, los ceñidores y los rhyta no están atestiguados como productos de Alašiya; al contrario que el lino.

Aunque la exportación más importante de Alašiya era el cobre, solo encontramos dos referencias a éste en los textos hititas: la tablilla KBo XII 38, ya mencionada, y la tablilla KBo 4.1. Ésta última pertenece a un ritual mágico, en la que se enumera cada uno de los materiales depositados sobre los cimientos de una nueva casa, entre los que se encuentran cobre procedente del Monte Taggata ${ }^{39}$, en Alašiya.

Otros dos textos de inventario, IBoT 1.31 y KBo 18.175 (CTH 241.2), mencionan el lino de Alašiya ${ }^{40}$. Vigo ${ }^{41}$ atestiguó que Alašiya no contaba con una producción propia de lino, sino que éste se adquiría en Egipto y posteriormente se convertía, en la isla, en un

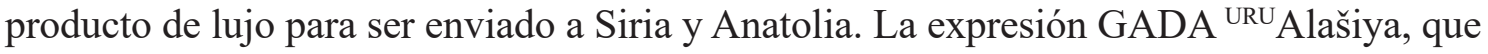
aparece en los textos de inventario hitita, traducida por Vigo como "lino Chipriota", hace, probablemente, referencia al modo en que ha sido elaborado y al lugar de su producción, y no al origen de éste.

Un texto médico (KUB 8.38+KUB 44.63 III) menciona una substancia llamada maruwašha, traída desde Alašiya ${ }^{42}$. Esta substancia, que podría ser malaquita verde ${ }^{43} \mathrm{O}$

31 de Martino, 2007: 483.

${ }^{32}$ Kitchen y Lawrence, 2012: 654.

33 de Martino, 2007: 483.

${ }^{34}$ Kitchen y Lawrence, 2012b:182-182.

35 de Martino, 2007: 483.

36 Otten, 1963: 10-13; de Martino, 2008: 249.

37 Knapp, 1980.

${ }^{38}$ Knapp (1980: 46) teoriza que la existencia de este tipo de parafernalia, implicaría la existencia de équidos en la isla.

${ }^{39}$ Hellbing (1979: 55) cree que la Montaña Tagatta se correspondería al sitio chipriota de Tamassos, localizado al sureste de la isla.

${ }^{40}$ En total se registran 37 piezas de lino en IBoT 1.31 pbv. 2-4, y 5 camisas de lino en KBo 18.175 i 5.

${ }^{41}$ Vigo 2010: 293.

${ }^{42}$ Singer y Gestoso Singer, 2014: 318; Hellbing 1979, 55.

${ }^{43}$ Singer y Gestoso Singer, 2014: 318-320. 
azurita $^{44}$ en polvo, puede que estuviese relacionada con una cura para el mal que aquejaba los ojos del rey Hattušili III, enviada por el faraón Ramsés II ${ }^{45}$.

El reino chipriota además aparece mencionado en otros dos textos de encantaciones: KUB XV 34 I 58 y KUB XV 35+ KBo II 9 I 33. El segundo texto es una plegaría a la diosa Ishtar de Nínive, a la que ruegan que vuelva, mencionando una serie de países en los que se encontraría Alašiya ${ }^{46}$.

Alašiya también se menciona también en dos oráculos hititas: KUB XXIV 30 obv. 24' y KBo XXXII 226 obv. 2; aunque debido al estado de las tablillas no se pueden extraer más datos ${ }^{47}$.

\section{Materiales hititas en Chipre}

En Chipre ProBA (1650-1400 a.C.), los grupos de élite adoptaron y asimilaron varios aspectos de tecnologías foráneas e iconografías. La hibridación de los motivos locales e importados y el simbolismo se muestra de forma aparente en la cultura material Pro BA. En el Pro BA 2 (LC IIA-IIC temprano), estas élites comienzan a utilizar o llevar objetos lujosos de oro o marfil y recipientes ceremoniales con forma de embudo muy similares a los encontrados en el Egeo y el Levante. Estos rhyta, junto con utensilios de oro, son mencionados como objetos de intercambio entre Chipre y la corte hitita en las tablillas en acadio de los ss. XIV y XIII a.C.. Varios objetos de oro, piedra y metal encontrados en Enkomi y decorados con esfinges, animales y jeroglíficos revelan un simbolismo cósmico y sugieren una asociación cercana con las ideologías políticas del Oriente Próximo ${ }^{48}$. Sin embargo, los materiales hititas encontrados en Chipre son muy escasos. Entre los materiales hallados se encuentran posibles sellos, figurillas y cerámica.

Los sellos de posible procedencia hitita son: un sello de oro en Tamassos, siendo éste el único que se puede atestiguar completamente como un hallazgo hitita ${ }^{49}$; un sello biconvexo; un sello en anillo de Hala Sultan Tepe; un sello cuadrado; un sello cónico en Enkomi; un sello cilíndrico en Kourion ; y una impronta de sello en Enkomi ${ }^{50}$.

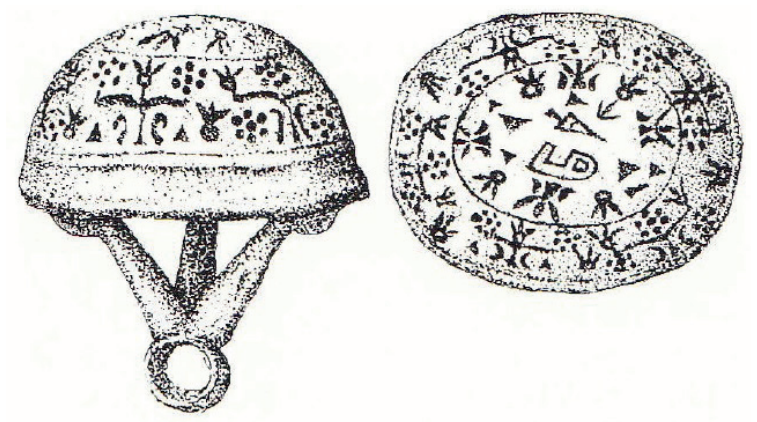

Figura 2: Sello de oro de Tamassos (Chipre). (Kozal, E, 2002, "Hethitische hethitisch beeinflußte Objekte aus Zypern”, p. 655.)

El sello biconvexo, de Hala Sultan Tepe, de piedra verde presenta jeroglíficos luvitas y una forma conocida de sello hitita. Mason ${ }^{51}$, sin embargo cree que este sello sería una

44 Beckman, 1996b: 35; de Martino 2008: 252.

${ }^{45}$ Es evidente que la medicina egipcia gozaba de una gran popularidad en la corte hitita y, como se puede comprobar en varios textos, como la tablilla KBo 28.30, el faraón egipcio aconsejaba y prestaba sus médicos al rey (Beckman, 1996: 131-132).

${ }^{46}$ Collins, 1997: 164-165.

47 de Martino 2008, 252.

48 Knapp, 2013: 428.

49 Kozal, 2002: 651.

${ }^{50}$ Kozal, 2002: 651

${ }^{51}$ Mason, 1964. 
imitación local de gran calidad, por lo que esta pieza solamente mostraría influencias hititas. Según Porada ${ }^{52}$, las influencias hititas también aparecen en sus motivos. Debido al motivo del disco solar alado, cree que este anillo-sello tiene un origen estilístico en Asia occidental, específicamente de la zona de Emar. El anillo de sello de Hala Sultan Tekke muestra solo una influencia hitita en el norte de Siria, desde donde llegaría a Chipre. Por lo tanto no habría una influencia directa en los estilos hititas de los sellos sobre Chipre.

Otros dos sellos procedentes de Enkomi y una impresión de sello procedentes de Kourion se han visto como un tipo de modelo antiguo hitita por Cook. Sin embargo J.C. Courtois y J. y E. Lagarce ${ }^{53}$ reconocen un estilo egipcio en la impresión del sello, en la forma en que se representan las plumas y la actitud de los dos pájaros muertos. Por lo tanto, no hay influencias hititas en esta pieza ${ }^{54}$. Cook también opina que el sello de Enkomi presenta motivos hititas; sin embargo, Kozal ${ }^{55}$ muestra que los motivos provienen del periodo del karum en Konya-Karahöyük, por lo que no puede tener un origen hitita ni influencias hititas.

Otros cuatro objetos en forma de figurillas ${ }^{56}$ parecen ser regalos diplomáticos, al ser considerados imágenes de culto, cuya producción y circulación estaba limitada a los circuitos oficiales. Estos son una figurilla de plata con una deidad que porta vestimenta típicamente hitita y un casco con cuernos, sobre un ciervo, de Kalavassos-Hagios Dhimitrios $^{57}$; una figurilla de bronce; la cabeza de una figura en terracota; y la cabeza de terracota de un toro, de procedencia hitita y hallada en la necrópolis de Agia Paraskevi, cerca de Nicosia ${ }^{58}$, datada en el s. XIII a.C.

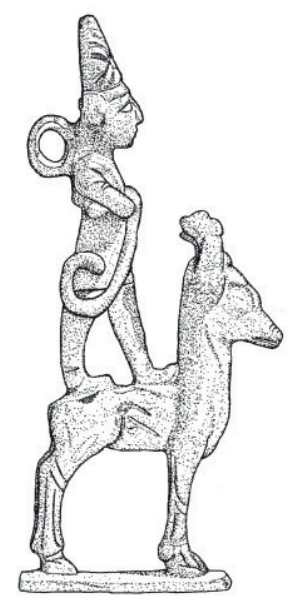

Figura 3: Figurilla de plata que representa a una deidad sobre un ciervo, hallada en Kalavassos-Hagios Demetrios (Chipre). (Genz, H., 2011, "Foreing Contacts..., p.308.)

La cerámica de posible origen hitita u anatolio encontrada en Chipre proviene de los yacimientos de Hagios Iakovos, Hala Sultan Tekke, Kition y Kazaphani. De todas ellas, solo se describió y publicó el fragmento de Hagios Iakovos y las conchas de Hala Sultan Tekke. Sin embargo, estas publicaciones solo las relacionan con la conquista hitita de Chipre y no mencionan si tienen un origen hitita o anatolio. Mientras que el fragmento de Hagios Iakovos no parece ser hitita, al sugerir Åström ${ }^{59}$ una procedencia Siria; las conchas

\footnotetext{
52 Porada, 1983: 219-220.

53 Courtois et al. 1986.

54 Kozal, 2002: 652

55 Kozal,2002: 652-653

56 Kozal, 2002: 653.

57 de Martino, 2008: 254.

58 Karageorghis $(1999,147-150)$ cree que contiene muchas similitudes con otra pieza hallada en Boğazköy.

59 Åström, 1972: 745.
} 
de Hala Sultan Tekke, fueron descritas por Ausgraber como anatolias y Mielke las sitúa en el suroeste de Anatolia, en donde encuentra parecidos con los hallados en Beycesultan ${ }^{60}$. Por lo tanto, debido a las escasas publicaciones y los datos ofrecidos sobre estas cerámicas, no se puede concluir que se haya realizado hasta el momento ningún hallazgo de cerámica hitita en Chipre ${ }^{61}$.

\section{Materiales chipriotas en Hatti}

Durante una excavación en la capital hitita en el año 1979 se encontró un fragmento, con un peso de siete quilos, de lingote de cobre de tipo de piel de buey, que fue identificado por Müller-Karpe ${ }^{62}$ como cobre procedente de Alašiya y datado entre los ss. XIII y XIV. Este descubrimiento, como se ha tratado anteriormente, podría considerarse un indicio del tributo en cobre que debía pagar a la corte hitita.

Además, también se han encontrado numerosos restos cerámicos de "Red Lustrous Wheel-made Ware" en Boğazköy ${ }^{63} \mathrm{y}$, especialmente, se encuentra presente en el sureste anatolio y en el interior del reino hitita. La arcilla de esta cerámica es muy homogénea y los colores varían del rojo ladrillo al rosa, ocasionalmente con una sombra gris en el centro. Las paredes son gruesas, normalmente de unos ocho milímetros., y están cubiertas con una pátina del mismo color y barnizadas con un brillo. Estos recipientes presentan varias formas, pero en Anatolia se encuentran estas cerámicas en forma de huso o en forma de brazo. Estos últimos se moldeaban probablemente con un palillo de madera y luego se ahuecaban con un objeto de forma cilíndrico ${ }^{64}$, y su función probablemente fuese ceremonial, al contener algún tipo de aceite.

Los fragmentos cerámicos hallados Hattuša se distribuyen tanto por la ciudad alta como por la ciudad baja; especialmente como relleno de los estanques de la ciudad alta, próximos a los templos, lo que sugiere que esta cerámica estaba destinada a las actividades relacionadas con el culto. Aunque los textos hititas no hacen mención en ningún momento a esta cerámica y tampoco adoptaron ningún ritual procedente de Alašiya; Mielke ${ }^{65}$ cree que estarían presentes en las ceremonias de los templos hititas y podrían destinarse a ungir con aceite a las estatuas o personas. En un extensivo estudio sobre la cerámica "Red Lustrous Wheel-made Ware" realizado por Knappett et al, ${ }^{66}$ se comprueba que una parte de esta cerámica en Boğazköy se trataba con cera de abeja, lo que probaría que contenían líquidos; aunque el estudio no puede aportar más datos acerca de si estas cerámicas eran tratadas antes, o después, de su llegada a la capital hitita.

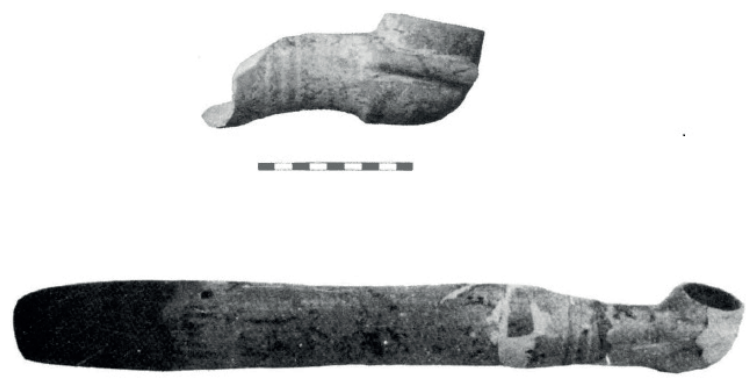

Figura 4: Cerámicas "Red Lustrous Wheel-made Ware" en forma de brazo, halladas en Boğazköy (superior) y Alaca Höyük. (Mielke, D.P., 2007, "Red Lustrous..., p. 157.)

\footnotetext{
60 Kozal, 2002: 653-654.

61 Kozal, 2002: 654.

${ }^{62}$ Müller-Karpe, 1980, 303-304.

63 Knappett et al., 2005: 33.

64 Sjöqvist, 1940: 51.

65 Mielke, 2007:163.

${ }^{66}$ Knappett et al., 2005: 48.
} 


\section{Conclusiones}

Para concluir, las relaciones entre los hititas y Alašiya parecen haber sido pacificas hasta la conquista de la isla por Tudhaliya IV. Hasta entonces está probado que no existirían conflictos entre ambos reinos, debido a la posibilidad de los opositores dinásticos hititas a encontrar un refugio en la isla y al comercio que se atestigua, gracias a las fuentes textuales y los datos arqueológicos.

No parece que los hititas conquistasen la isla hasta el reinado de Tudhaliya IV, a pesar de las demandas de Arnuwanda I. No solo el hecho de que suponga la primera conquista naval hitita, sino que su insistencia, al tener que ser conquistada dos veces, acrecienta la situación desesperada en la que se encontraba el reino de Hatti al final del Imperio. La urgencia, debido a las expansiones asirias y las invasiones de los Pueblos del Mar, sumados a varios años de continuas plagas y escasez, acrecentaron sin duda esta dependencia no sólo de los productos de la isla, sino también de aquellos que provenían de otros países del Mediterráneo oriental, como Egipto, y que recalarían en este reino y fueron, sin duda, los motivos principales que motivaron la conquista hitita sobre la isla. Además, aunque las conquistas de Tudaliya IV y Suppiluliuma II fuesen efectivas durante un corto periodo, y sometiesen a Alašiya a un tratado, en el que se establecía un tributo, parece que el reino seguía contando con una gran independencia, como se atestigua por la correspondencia con Ugarit.

A pesar de los escasos datos arqueológicos y textuales hallados, puede establecerse que existieron unas relaciones comerciales entre ambos reinos durante el Bronce Final; ya que, algunos de los bienes intercambiados entre ambos reinos, como aceites o, incluso, el cobre, ya que era frecuentemente reutilizado, no dejarían muchas trazas de su existencia en los yacimientos. Sin embargo, actualmente, las escasas evidencias arqueológicas no permiten conocer en qué medida existía un comercio entre ambos reinos, si éste se producía de forma regular o esporádicamente. Mientras que la mayor parte de estos materiales se corresponden a la cerámica chipriota, seguramente utilizada para transportar algún tipo de líquido, o aceite, destinado a las ceremonias cultuales; si atendemos a las fuentes textuales también puede atestiguarse la existencia un comercio textil y del cobre, y sus derivados, a pesar de que los datos arqueológicos no ofrecen, hasta ahora, suficientes datos que las apoyen.

\section{Bibliografía}

Åström, P., 1972, The Swedish Cyprus Expedition IV, IC, The Late Cypriot Bronze Age. Lund.

Beckman, G., 1996, Hittite Diplomatic Texts. Atlanta.

Beckman, G., 1996b, "Hittite Documents from Hattuša", in A.B. Knapp et al. (eds.), Sources for the History of Cyprus, vol 2. Near Eastern and Aegean texts from the third to the first millennia BC, Altamont, pp. 31-35.

Beckman et al., 2011, The Ahhiyawa Texts. Atlanta.

Bryce, T., 2005, The Kingdom of the Hittites (New Edition). Oxford.

Bryce, T., 2011, "The Hittite Deal with the Hiyawa-Men.", in Y. Cohen et al. (eds.), Pax Hethitica:Studies on the Hittites and their Neighbours in Honour of Itamar Singer, Wiesbaden, pp. 47-53.

Bryce, T. y Birkett-Rees, J., 2016, Atlas from the Ancient Near East. From Prehistoric to the Roman Imperial Period. London-New York.

Carruba, O., 1995, “Ahhiya e Ahhiyawa, la Grecia e l'Egeo”, in Th.P.J. van den Hout y J. de Roos (eds.), Studio Historiae Ardens: Ancient Near Eastern Studies Presented to Philo H.J. Houwink ten Cate on the Occasion of his 65th Birthday, Istanbul, pp. 7-21. 
Collins, B. J., 1997, "Ritual and Prayer to Ishtar of Ninveh (1.65) )", in W. Hallo (ed.), The Context of Scripture, Vol. 1, Leiden-New York-Köln, pp. 164-165.

Collins, B.J., 2007, The Hittites and their World. Atlanta.

Courtois, J-C. et al., 1986, Enkomi et le Bronze Récent à Chipre. Nicosie.

de Martino, S., 2007, "Il trattato tra Hatti e Alašiya, KBo XII 39", in M. Alparslan et al. (eds.), VITA. Festschriff in Honor of Belkis Dinçol and Ali Dinçol, Istanbul, pp. 483492.

de Martino, S., 2008, "Relations between Hatti and Alašiya according to Textual and Archaeological Evidence", in G. Wilhelm (ed.). Hattuša-Boğazkoy. Das Hethiterreich im Spannungsfeld des Alten Orients, Wiesbaden, pp. 247-163.

Genz, H., 2011, "Foreing Contacts of the Hittites (Chapter 12)", in H. Genz y P. D. Mielke (eds.), Insights into Hittite History and Archaeology, Leuven-Paris-Walpole, pp. 301-331.

Goren et al., 2003, "The location of Alashiya: New Evidences from Petrographic Investigation of Alashiya Tablets from El-Amarna and Ugarit", American Journal of Archaeology, 107-2, pp. 233-255.

Güterbock, H. G., 1967, “The Hittite Conquest of Cyprus Reconsidered”, Journal of Near Eastern Studies, Vol.6, 2, pp. 73-81.

Hawkins, J. D., 1995, The hieroglyphic inscription of the sacred pool complex at Hattusa (Südburg). Wiesbaden.

Hellbing, L, 1979, Alasia Problems. Götenborg.

Heltzer, M., 2001, "Asylum on Alashia (Cyprus)", Zeitschrift fur Altorientalische und Biblische Rechtgeschichte, 7, Wiesbaden, pp.268-273.

Hoffner, H. A. Jr, 1997, "The Hittite Conquest of Cyprus: two Inscriptions of Suppiluliuma II (1.75)", in W. Hallo (ed.), The Context of Scripture, Vol. 1, Leiden-New York- Köln, pp.192-193.

Hoffner, H. A. Jr, 2009, Letters form the Hittite Kingdom. Leiden.

Houwink ten Cate, Ph. H.J., 1983-1984, "Sidelights on the Ahhiyawa-Question from Hittite Vassal and Royal Correspondence", Jaarbericht ex Oriente Lux 28, pp. 33-79.

Karageorghis, V., 1999, "An anatolian terracotta bull's head from the Late Cypriot Necropolis of Agia Paraskevi", Report of the Deparment of Antiquities Cyprus, pp. 147150 .

Kelder, J., 2004-2005, "Mycenaeans in Western Anatolia", in J.P. Stronk y M.D. de Weerd, Talanta XXXVI-XXXVII (2004-2005). Proceedings of the Dutch Archaeology and Historical Society, Vols. XXXVI-XXXVII, pp.49-86.

Keswani, P. S., 1996, "Hierarchies, Heterarchies and Urbanization Processes: The View from Bronze Age Cyprus", Journal of Mediterranean Archaeology 9, pp. 211-250.

Kitchen, K.A. y Lawrence, P. J. N., 2012, Treaty, Law and Covenant in the Ancient Near East. Part 1: The Texts. Wiesbaden.

Kitchen, K.A. y Lawrence, P. J. N., 2012b, Treaty, Law and Covenant in the Ancient Near East. Part 3: Overall Historical Survey. Wiesbaden.

Klengel, H, 1999, Geschichte des hethitischen Reiches. Leiden.

Knapp, A. B., 1980, "KBo 1 26: Alasiya and Hatti”, Journal of Cuneiform Studies, Vol. 32, 1, pp. 43-47.

Knapp, A. B., 1988, “Hoards D'Oeuvres: Of Metals and Men on Bronze Age Cyprus", Oxford Journal of Archaeology 7, pp. 147-176.

Knapp, A. B., 2008, Prehistoric and Protohistoric Cyprus. Identity, Insularity, and Connectivity. Oxford-New York.

Knapp, A. B., 2013, The Archaeology of Cyprus. From Earliest Prehistory through the Bronze Age. New York. 
Knappett, C. et al., 2005, "The Circulation and Consumption of Red Lustrous Wheelmade Ware: Petrographic, Chemical and Residue Analysis", Anatolian Studies 55, pp. 25-59.

Kozal, E., 2002, "Hethitische hethitisch beeinflußte Objekte aus Zypern", in R. Aslan et al. (eds.), Mauerschau, Festschrift für Manfred Korfmann, Vol. 2, RemshaldenGrunbach, pp. 651-661.

Mason, O., 1964, "Kypriaka Recherches sur le antiquités de de Tamassos", Bulletin de correspondance hellénique 88, pp. 199-238.

Mielke, D.P., 2007, "Red Lustrous Wheelmade Ware from Hittite Contexts", in I. Hein (ed.), The Lustrous Wares of Late Bronze Age Cyprus and the Eastern Mediterranean. Papers of a Conference, Vienna 5th-6th of November 2004. Contributions to the Chronology of the Eastern Mediterranean XIII, Wien, pp.155-168.

Muhly, J.D., 1972, "The land of Alashiya: References to Alashiya in the texts of the Second Millennium B.C. and the History of Cyprus in the Late Bronze Age", in Proceedings of the First International Cyprological Congress, Nicosia, pp. 201-19.

Müller-Karpe, A., 1980, "Die Ausgrabungen in Boğazköy-Hattuša 1979 - Die Funde", Archäologischer Anzeiger, pp. 303-307.

Otten, H., 1963, "Neue Quellen zum Ausklang des Hethitischen Reiches", Mitteilungen der Deutschen Orient-Gesellschaft zu Berlin 94, pp. 1-23.

Porada, E., 1983, “A Seal Ring and two seals from Hala Sultan Tekke”, in P. Åström et al. (eds.), Sultan Tekke, 8 Excavation 1971-799, Göteborg, pp. 219-220.

Schaeffer, C.,1952, Enkomi- Alasia. Nouvelles missions en Chypre 1946-1950, Paris. Siegelová, J. y Tsumoto, H., 2011, "Metals and Metallurgy in Hittite Anatolia", in H. Genz y D. P. Mielke (eds.), Insights into Hittite History and Archaology, Leuven-ParisWalpole, pp 275-300.

Singer, I. y Gestoso Singer, G., 2014, "Alašian products in Hittite sources ", in Z. Csabai y T. Grüll (eds.), Studies in Economic and Social History of the Ancient Near East in Memory of Péter Vargyas- Ancient Near East and Meditteranean Studies, Vol. 2, Pécs, pp.317-336.

Sjöqvist, E., 1940, Problems of the late Cypriote Bronze Age : the Swedish Cyprus expedition, Stockholm.

Steel, L., 2004, Cyprus Before History. From the Earliest Settlers to the End of the Bronze Age. London.

Stefanini, R., 1964-1965, Una lettera della regina Puduhepa al re di Alasija (KUB XXI 38). Firenze.

Ünal, A., 1991, "Two Peoples on Both Sides of the Aegean Sea. Did the Achaeans and the Hittites Know Each Other?", in H.I.H. Prince Takahito Mikasa (ed.), Essays on Ancient Anatolian and Syrian Studies in the 2nd and 1st Millennium B. C., Wiesbaden, pp. $16-44$.

van den Hout, Th., 2002, “The Divine Stone-House Reconsidered”, in K.A. Yener y H. A. Hoffner Jr. (eds.), Recent Developments in Hittite Archaeology and History, Winona Lake, pp. 73-91.

Vigo, M., 2010, "Linen in Hittite Inventory Texts", en C. Michel y M.-L. Nosch (eds.), Textile Terminologies in the Ancient Near East and Mediterranean from the Third to the First Millenia B.C., Oxford, pp. 290-322.

Webb, J.M. 1999, Ritual Architecture, Iconography and Practice in the Late Cypriote Bronze Age. Jonsered: Paul Forlag Astroms. 


\author{
Abreviaturas \\ CTH Laroche, E., 1971, Catalogue des textes hittites. Paris. \\ EA Correspondencia de Amarna \\ IBoT Istanbul Arkeoloji Müzelerinde Bulunan Boğazköy Tabletleri \\ KBo Keilschrifttexte aus Boghazköi \\ KUB Keilschrifturkunden aus Boghazköi \\ RS Ras Shamra
}

